\title{
Synthesis, characterization and biological evaluation of 2,5-di-substituted 1,3,4-oxadiazole derivatives
}

\author{
Hitesh Makwana, Yogesh T. Naliapara* \\ Department of Chemistry, Saurashtra University, Rajkot, Gujarat, India \\ *E-mail address: naliaparachem@yahoo.co.in
}

\begin{abstract}
We have reported some novel 1,3,4-oxadiazole synthesized by conventional method .The reaction of 5-bromothiophene-2-carbohydrazide and different benzoic acid derivatives reflux in toluene using phosphorus oxychloride as a catalyst, yielded a series of 2,5-di-substituted 1,3,4oxadiazole HM-2a to HM-2t. The newly synthesized 2,5-di-substituted 1,3,4-oxadiazole were purified by column chromatography and characterized by IR, Mass, 1H NMR, 13C NMR spectroscopy and elemental analysis. All synthesized compounds were screened for antimicrobial activity using cup plate method. All the compounds showed moderate to good antimicrobial activity and anti fungal activity.
\end{abstract}

Keywords: Substituted 1,3,4-oxadiazoles; phosphoric anhydride; antimicrobial activity

\section{INTRODUCTION}

In the field of synthetic organic chemistry, major challenges are to develop the new method for the synthesis of five member heterocyclic compounds. Literature survey reveals five member 1,3,4-oxadiazole derivatives, which belong to an important group of heterocyclic compounds, have been the issue of wide-ranging study in the recent time. Various reports have displayed their chemistry and use [1-3]. A Wide variety of substituted 1,3,4-oxadiazoles have attracted considerable attention in the field of drug discovery because of their wide range of pharmacological activities, including antiproliferative [4], antifungal [5], antibacterial [6,7], anticancer [8], anti-tubercular [9], GABAA receptor agonists [10], anti-inflammatory [11], anti HIV [12]. Different methods have been reported for the synthesis of 1,3,4-oxadiazoles involving cyclization of 1,4-di-substituted thiosemicarbazide in the presence dicyclohexylcarbodiimide (DCC) [13]. Several cyclodehydrating agents such as $\mathrm{Et}_{2} \mathrm{O}-\mathrm{BF}_{3}$, triflic anhydride, thionyl chloride, polyphosphoric acid, 1,1,1,3,3,3hexamethyldisilazane, sulfuric acid and phosphorus oxychloride [14] have been used. However, use of phosporic anhydried is better than phosphorous oxychloride [15]. 


\section{EXPERIMENTAL}

All chemicals and solvents were purchased from Spectrochem Pvt Ltd., Mumbai of AR grade and were used without further purification. Melting points were taken in open capillary method and are uncorrected. IR spectra were recorded on FTIR-8400 spectrophotometer (Shimadzu, Kyoto, Japan), using DRS probe KBr pallet. ${ }^{1} \mathrm{H}-\mathrm{NMR}$ spectra of the synthesized compounds were recorded on a Bruker-Avance-II (400 MHz) DMSO- $d 6$ solvent. Chemical shifts are expressed in $\delta$ ppm downfield from TMS as an internal standard. Mass spectra were determined using direct inlet probe on a GCMS-QP 2010 mass spectrometer (Shimadzu, Kyoto, Japan). Physical constants of the synthesized compounds are shown in Table 1.

\section{1. General synthesis of methyl 5-bromothiophene-2-carboxylate (Int 1)}

To a well stirred 5-bromothiophene-2-carboxylic acid $(0.01 \mathrm{~mol})$ in methanol $(25 \mathrm{ml})$, add concentrated sulphuric acid $(0.01 \mathrm{~mol})$ at $0-15^{\circ} \mathrm{C}$ and then reflux the reaction mass at 65 $70{ }^{\circ} \mathrm{C}$ for 6-7 hours. Then after completion of the reaction cool the reaction mixture at $15^{\circ} \mathrm{C}$ and add cold water and stir for 3 hours at room temperature. Separated solid washed with water and collected by filtration.

\section{2. General synthesis of 5-bromothiophene-2-carbohydrazide (Int 2)}

Methyl 5-bromothiophene-2-carboxylate $(0.01 \mathrm{~mol})$ in methanol was refluxed with hydrazine hydrate $(0.1 \mathrm{~mol})$ for 5 to 6 hours. After completion of the reaction checked by TLC, the reaction mixture was cooled to room temperature. The separated solid was filtered, was with cold methanol and crystallized from methanol.

\section{3. General synthesis of Oxadiazole (Int HM-2a-t)}

A mixture of 5-bromothiophene-2-carbohydrazide $(2.17 \mathrm{~g}, 0.01 \mathrm{~mol})$ and different aryl acids $(0.01 \mathrm{~mol})$ in phosphorous oxychloride $(10 \mathrm{ml})$, was refluxed with continuous stirring. After completion the reaction (6-7 hour monitoring by TLC), the content was cooled to room temperature then add ice cooled water and neutralized with sodium

\section{REACTION SCHEME}<smiles>COC(=O)c1ccc(Br)s1</smiles>

Scheme 1. (a) Methanol, sulfuric acid, $65^{\circ} \mathrm{C}$ (b) Hydrazine Hydrate, $60{ }^{\circ} \mathrm{C}$ (c) $\mathrm{POCl}_{3}$, Reflu. 
<smiles>[R]c1ccc(-c2nnc(-c3ccc(Br)s3)o2)cc1</smiles>

Scheme 1(continue). (a) Methanol, sulfuric acid, $65^{\circ} \mathrm{C}$ (b) Hydrazine Hydrate, $60{ }^{\circ} \mathrm{C}(\mathrm{c}) \mathrm{POCl}_{3}$, Reflu.

Table 1. Synthesis of oxadiazoles.

\begin{tabular}{|c|c|c|c|c|}
\hline Entry & $\mathbf{R}$ & Time h & Yield (\%) & mp. ${ }^{\circ} \mathrm{C}$ \\
\hline HM-2a & $-\mathrm{C}_{6} \mathrm{H}_{5}$ & 6.5 & 81 & $182-184$ \\
\hline $\mathrm{HM}-2 \mathrm{~b}$ & 5- $\mathrm{Cl} \mathrm{C} \mathrm{C}_{4} \mathrm{H}_{2}$ & 5.5 & 79 & $165-167$ \\
\hline $\mathrm{HM}-2 \mathrm{c}$ & 5- $\mathrm{Br} \mathrm{C}_{4} \mathrm{H}_{2}$ & 6 & 87 & $172-174$ \\
\hline HM-2d & $\mathrm{CH}_{2}-\left(4-\mathrm{OMe} \mathrm{C}_{6} \mathrm{H}_{4}\right)$ & 7 & 65 & 194-196 \\
\hline HM-2e & $4-\mathrm{F} \mathrm{C}_{6} \mathrm{H}_{4}$ & 6.5 & 72 & $218-220$ \\
\hline HM-2f & $\mathrm{CH}_{2}-\left(2,6-\mathrm{di}-\mathrm{Cl} \mathrm{C}_{6} \mathrm{H}_{3}\right)$ & 6 & 77 & 206-208 \\
\hline HM-2g & $4-\mathrm{OMe} \mathrm{C}_{6} \mathrm{H}_{4}$ & 6 & 84 & $166-168$ \\
\hline HM-2h & $3-\mathrm{Cl} \mathrm{C} \mathrm{H}_{4}$ & 6.5 & 81 & $178-180$ \\
\hline HM-2i & $3-\mathrm{NO}_{2} \mathrm{C}_{6} \mathrm{H}_{4}$ & 7 & 76 & 192-194 \\
\hline $\mathrm{HM}-2 \mathrm{j}$ & $2-\mathrm{Cl} \mathrm{C} \mathrm{H}_{4}$ & 6.5 & 78 & $156-158$ \\
\hline HM-2k & $4-\mathrm{NO}_{2} \mathrm{C}_{6} \mathrm{H}_{4}$ & 6 & 85 & $202-204$ \\
\hline HM-21 & $2-\mathrm{OH} \mathrm{C} \mathrm{C}_{4}$ & 7 & 88 & $214-216$ \\
\hline HM-2m & 4- $\mathrm{NH}_{2} \mathrm{C}_{6} \mathrm{H}_{4}$ & 5.5 & 87 & $232-234$ \\
\hline HM-2n & 2-Cl-4- $\mathrm{NO}_{2} \mathrm{C}_{6} \mathrm{H}_{3}$ & 6 & 75 & 196-198 \\
\hline HM-2o & 2-OEt $\mathrm{C}_{6} \mathrm{H}_{4}$ & 6 & 71 & $208-210$ \\
\hline HM- $2 p$ & 2,4-di-Cl C ${ }_{6} \mathrm{H}_{3}$ & 7 & 83 & $188-190$ \\
\hline HM-2q & $4-\mathrm{OH} \mathrm{C} \mathrm{C}_{6}$ & 6.5 & 87 & 196-198 \\
\hline HM-2r & $3,5-\mathrm{di}-\mathrm{NO}_{2} \mathrm{C}_{6} \mathrm{H}_{3}$ & 5.5 & 89 & $220-222$ \\
\hline HM-2s & $2,5-\mathrm{di}-\mathrm{NO}_{2} \mathrm{C}_{6} \mathrm{H}_{3}$ & 6 & 77 & $238-240$ \\
\hline HM-2t & 2-Me $\mathrm{C}_{6} \mathrm{H}_{4}$ & 6.5 & 81 & $168-170$ \\
\hline
\end{tabular}




\section{SPECTRAL DATA OF THE SYNTHESIZED COMPOUNDS}

\section{2-(5-bromothiophen-2-yl)-5-phenyl-1,3,4-0xadiazole (HM-2a):}

Yellow solid; $\mathrm{mp} 182-182{ }^{\circ} \mathrm{C} ; \mathrm{R}_{\mathrm{f}}$ 0.52 (4:6 EtOAc-toluene, acetic acid 1 drop); IR ( $\left.\mathrm{KBr}\right)$ : 3093, 3063, 2906, 2349, 1799, 1697, 1641, 1593, 1548, 1489, 1425, 1274, 1209, 1066, 1033, 968. 819, 771, 688, $563 \mathrm{~cm}^{-1}$; ${ }^{1} \mathrm{H}$ NMR: $\delta$ 7.096-7.106 (d, 1H, -CH thiophene ring, $\left.j=4 \mathrm{~Hz}\right)$, 7.476-7.507 (m, 3H, Ar-H phenyl ring ), 7.513-7.523 (d, $-\mathrm{CH}$, Ar-H thiophene ring, $j=4 \mathrm{~Hz}$ ), 8.027-8.051 (dd, 2H, Ar-H, phenyl ring, $j=1.6,7.6 \mathrm{~Hz}$ ). $\mathrm{MS}(\mathrm{m} / \mathrm{z}): 307\left(\mathrm{M}^{+}\right)$; Anal. Calcd for $\mathrm{C}_{12} \mathrm{H}_{7} \mathrm{BrN}_{2} \mathrm{OS}$ : C, 44.59; H, 2.18; N, 8.67; Found: C, 43.12; H, 3.21; N, 9.31.

\section{2-(5-bromothiophen-2-yl)-5-(5-chlorothiophen-2-yl)-1,3,4-thiadiazole (HM-2b):}

Yellow solid; mp 165-167 ${ }^{\circ}$; $\mathrm{R}_{\mathrm{f}} 0.46$ (4:6 EtOAc-toluene, acetic acid 1 drop); IR (KBr): 3269, 3095, 3014, 2924, 2364, 1805, 1647, 1587, 1496, 1431, 1309, 1211, 1111, 1049, 1030, 968, 937, 802, 719, 663, $559 \mathrm{~cm}^{-1}$; ${ }^{1} \mathrm{H}$ NMR: $\delta$ 6.950-6.960 (d, 1H, -CH, Ar-H, $\left.j=4 \mathrm{~Hz}\right)$, 7.087-7.096(d, $1 \mathrm{H}$, Ar-H, $j=3.6 \mathrm{~Hz}), 7.478-7.488(\mathrm{~d}, 1 \mathrm{H}, \mathrm{Ar}-\mathrm{H}, j=4 \mathrm{~Hz}), 7.512-7.522(\mathrm{~d}$, $1 \mathrm{H}, \operatorname{Ar}-\mathrm{H}, j=4 \mathrm{~Hz})$. MS $(\mathrm{m} / \mathrm{z}): 347\left(\mathrm{M}^{+}\right)$; Anal. Calcd for $\mathrm{C}_{10} \mathrm{H}_{4} \mathrm{BrClN}_{2} \mathrm{OS}_{2}: \mathrm{C}, 34.55 ; \mathrm{H}$, 1.16 ; N, 8.06; Found: C, 36.27; H, 2.23; N, 7.37.

\section{2,5-bis(5-bromothiophen-2-yl)-1,3,4-oxadiazole (HM-2c):}

Yellow solid; mp 172-174 ${ }^{\circ} \mathrm{C}$; $\mathrm{R}_{\mathrm{f}} 0.51$ (4:6 EtOAc-toluene, acetic acid 1 drop); IR (KBr): 3211, 3061, 2981, 2328, 1812, 1634, 1574, 1482, 1427, 1334, 1247, 1141, 1024, 1065, 936, 883, 752, 675, $567 \mathrm{~cm}^{-1}$; MS (m/z): $392\left(\mathrm{M}^{+}\right)$; Anal. Calcd for $\mathrm{C}_{10} \mathrm{H}_{4} \mathrm{Br}_{2} \mathrm{~N}_{2} \mathrm{OS}_{2}: \mathrm{C}, 30.63 ; \mathrm{H}$, 1.03 ; N, 7.14; Found: C, 32.18; H, 2.24; N, 6.76.

2-(5-bromothiophen-2-yl)-5-(4-methoxybenzyl)-1,3,4-oxadiazole (HM-2d):

Yellow solid; mp 194-196 ${ }^{\circ} \mathrm{C}$; $\mathrm{R}_{\mathrm{f}} 0.42$ (4:6 EtOAc-toluene, acetic acid 1 drop); IR (KBr): 3298, 3087, 2976, 2347, 1867, 1678, 1597, 1443, 1401, 1318, 1279, 1132, 1037, 1008, 967, 864, 789, 668, $587 \mathrm{~cm}^{-1}$; MS $(\mathrm{m} / z): 351\left(\mathrm{M}^{+}\right)$; Anal. Calcd for $\mathrm{C}_{10} \mathrm{H}_{4} \mathrm{Br}_{2} \mathrm{~N}_{2} \mathrm{OS}_{2}: \mathrm{C}, 47.88 ; \mathrm{H}$, 3.16 ; N, 7.98; Found: C, 45.24; H, 2.47; N, 8.24.

2-(5-bromothiophen-2-yl)-5-(4-fluorophenyl)-1,3,4-oxadiazole (HM-2e): Yellow solid; mp 218-220 ${ }^{\circ} \mathrm{C}$; $\mathrm{R}_{\mathrm{f}} 0.57$ (4:6 EtOAc-toluene, acetic acid 1 drop); IR (KBr): 3284, 3067, 3021, 2931, 2355, 1812, 1652, 1591, 1483, 1425, 1311, 1249, 1171, 1064, 1014, 991, 941, 845, 762, 647, $587 \mathrm{~cm}-1$; MS (m/z): $325\left(\mathrm{M}^{+}\right)$; Anal. Calcd for $\mathrm{C}_{10} \mathrm{H}_{4} \mathrm{BrClN}_{2} \mathrm{OS}_{2}: \mathrm{C}, 44.33$; H, 1.86; N, 8.62; Found: C, 47.24; H, 2.78; N, 7.86.

\section{2-(5-bromothiophen-2-yl)-5-(2,6-dichlorobenzyl)-1,3,4-oxadiazole (HM-2f):}

Yellow solid; $\mathrm{mp} 182-182{ }^{\circ} \mathrm{C} ; \mathrm{R}_{\mathrm{f}} 0.52$ (4:6 EtOAc-toluene, acetic acid 1 drop); IR (KBr): 3107, 3078, 2925, 2384, 1764, 1679, 1627, 1568, 1555, 1447, 1407, 1292, 1224, 1078, 1039, 982. 832, 786, 674, $587 \mathrm{~cm}^{-1}$; MS $(\mathrm{m} / \mathrm{z}): 390\left(\mathrm{M}^{+}\right)$; Anal. Calcd for $\mathrm{C}_{13} \mathrm{H}_{7} \mathrm{BrCl}_{2} \mathrm{~N}_{2} \mathrm{OS}: \mathrm{C}$, 40.03; H, 1.81; N, 7.18; Found: C, 42.21; H, 3.04; N, 8.42.

\section{2-(5-bromothiophen-2-yl)-5-(4-methoxyphenyl)-1,3,4-oxadiazole (HM-2g):}

Yellow solid; $\mathrm{mp} 166-168{ }^{\circ} \mathrm{C}$; $\mathrm{R}_{\mathrm{f}} 0.53$ (4:6 EtOAc-toluene, acetic acid 1 drop); IR (KBr): 3045, 3081, 2934, 2375, 1776, 1683, 1645, 1579, 1564, 1434, 1431, 1279, 1231, 1067, 1021, 974. 843, 778, 681, $563 \mathrm{~cm}^{-1}$; MS $(\mathrm{m} / z): 337\left(\mathrm{M}^{+}\right)$; Anal. Calcd for $\mathrm{C}_{13} \mathrm{H}_{9} \mathrm{BrN}_{2} \mathrm{O}_{2} \mathrm{~S}: \mathrm{C}, 46.31$; H, 2.69; N, 8.31; Found: C, 48.24; H, 3.71; N, 7.14. 


\section{BIOLOGICAL ACTIVITY}

\section{1. Antimicrobial Sensitivity Testing}

\section{1. 1. Well Diffusion/Agar Cup Method}

In vitro affectivity of antimicrobial agents can be demonstrated by observing their capacity to inhibit bacterial growth on suitable media. The production of a zone depends on two factors namely bacterial growth and concentration of antimicrobial agent. The hole/well punch method was first used by Bennett. This diffusion method has proved more effective than many other methods. According to Lt. General Raghunath the well technique is 5-6 times more sensitive than using disk method.

\section{1. 2. Principle}

When antimicrobial substance is added in agar cup (made in a medium previously inoculated with test organism) the redial diffusion of an antimicrobial agent through the agar, produces a concentration gradient. The test organism is inhibited at the minimum inhibitory concentration (MIC), giving rise to a clear zone of inhibition.

Table 2. Antimicrobial Sensitivity Assay.

\begin{tabular}{|c|c|c|c|c|c|c|c|c|}
\hline \multirow{2}{*}{\begin{tabular}{c}
\multirow{2}{*}{$\begin{array}{c}\text { Sr. } \\
\text { No. }\end{array}$} \\
\multirow{2}{*}{ Code no. }
\end{tabular}} & \multicolumn{7}{|c|}{ antibacterial activity } & \multicolumn{2}{c|}{ antifungal activity } \\
\cline { 3 - 9 } & & $\begin{array}{c}E . \\
\text { coli }\end{array}$ & $\begin{array}{c}\text { P. } \\
\text { aeruginosa }\end{array}$ & $\begin{array}{c}\text { S. } \\
\text { aureus }\end{array}$ & $\begin{array}{c}\text { S. } \\
\text { pyogenus }\end{array}$ & $\begin{array}{c}\text { C. } \\
\text { albicans }\end{array}$ & $\begin{array}{c}\text { A. } \\
\text { niger }\end{array}$ & $\begin{array}{c}\text { A. } \\
\text { clavatus }\end{array}$ \\
\hline 1 & HM-2a & 250 & 250 & 500 & 500 & 500 & 250 & $>1000$ \\
\hline 2 & HM-2b & 500 & 500 & 250 & 500 & 250 & 500 & 250 \\
\hline 3 & HM-2c & 250 & 500 & 250 & 500 & 500 & 250 & 1000 \\
\hline 4 & HM-2d & 100 & 500 & 250 & 500 & 500 & 250 & $>1000$ \\
\hline 5 & HM-2e & 250 & 500 & 250 & 500 & 500 & 500 & 500 \\
\hline 6 & HM-2f & 500 & 200 & 500 & 250 & 250 & 500 & $>1000$ \\
\hline 7 & HM-2g & 250 & 500 & 250 & 500 & 500 & 1000 & 500 \\
\hline 8 & HM-2h & 100 & 250 & 200 & 125 & 200 & 250 & 250 \\
\hline 9 & HM-2i & 500 & 500 & 250 & 250 & 500 & 250 & 500 \\
\hline 10 & HM-2j & 250 & 250 & 500 & 250 & 100 & 100 & 100 \\
\hline 11 & HM-2k & 500 & 250 & 250 & 500 & 200 & 500 & 500 \\
\hline 12 & HM-2l & 250 & 250 & 500 & 250 & 250 & 100 & 250 \\
\hline 13 & HM-2m & 100 & 200 & 500 & 125 & 100 & 250 & 250 \\
\hline
\end{tabular}




\begin{tabular}{|c|c|c|c|c|c|c|c|c|}
\hline 14 & HM -2n & 250 & 200 & 500 & 250 & 500 & 250 & 500 \\
\hline 15 & HM -2o & 250 & 500 & 250 & 500 & 1000 & 500 & 500 \\
\hline 16 & HM -2p & 500 & 250 & 500 & 250 & 500 & 250 & 500 \\
\hline 17 & HM -2q & 250 & 500 & 500 & 200 & 250 & 1000 & $>1000$ \\
\hline 18 & HM -2r & 100 & 200 & 250 & 125 & 100 & 250 & 250 \\
\hline 19 & HM -2s & 500 & 250 & 500 & 250 & 250 & 500 & 1000 \\
\hline 20 & HM -2t & 100 & 250 & 200 & 125 & 100 & 250 & 250 \\
\hline Gentamycin & 0.05 & 1 & 0.25 & 0.5 & - & - & - \\
\hline \multicolumn{2}{|c|}{ Ampicilin } & 100 & 100 & 250 & 100 & - & - & - \\
\hline \multicolumn{2}{|c|}{ Chloramphenicol } & 50 & 50 & 50 & 50 & - & - & - \\
\hline \multicolumn{2}{|c|}{ Ciprofloxacin } & 25 & 25 & 50 & 50 & - & - & - \\
\hline \multicolumn{2}{|c|}{ Norfloxacin } & 10 & 10 & 10 & 10 & - & - & - \\
\hline \multicolumn{2}{|c|}{ Nystatin } & - & - & - & - & 100 & 100 & 100 \\
\hline \multicolumn{2}{|c|}{ Greseofulvin } & - & - & - & - & 500 & 100 & 100 \\
\hline
\end{tabular}

\section{CONCLUSION}

We have developed a novel 2,5-disubstituted-oxadiazole by the reaction of different aromatic acid and hydrazide in high yield and purity. The reaction of various aromatic acid and 5-bromothiophene-2-carbohydrazide was carried out by refluxing in $\mathrm{POCl}_{3}$. All the synthesized compounds were evaluated for their antimicrobial activity. The investigation of antibacterial and antifungal screening data revealed that, the compounds HM-2h, HM- $2 \mathrm{~m}$, HM-2r, and HM-2t shows good activity against bacterial stain, while HM-2h, HM- $\mathrm{j}$, HM$2 \mathrm{k}, \mathrm{HM}-2 \mathrm{r}$, and HM-2t shows comparatively good activity against fungal stain.

\section{Acknowledgements}

Authors are thankful to UGC, New Delhi for the financial support. 


\section{Referances}

[1] Potts, K. In Compr. Heterocyclic Chem.; Katritzky, A.R., Rees, Ch., Eds.; Pergamon Press: NY; 6 (1984) 427.

[2] Kulkarni Y. D., Rowhani A., J. Indian Chem. Soc. 66 (1989) 492.

[3] Mohamed Ashraf Ali, Mohammad Shaharyar, Bioorganic \& Medicinal Chemistry Letters 17 (2007) 3314-3316.

[4] Neelam Jain, D. P. Pathak, Pradeep Mishra, Sandeep Jain, Der Pharmacia Lettre, 5 (2013) 415-418.

[5] S. L. Gaonkar, K. M. L. Rai, B. Prabhuswamy, Eur. J. Med. Chem. 41 (2006) 841-846.

[6] Zampieri D., et al., Bioorg. Med. Chem. 17 (2009) 4693-4707.

[7] B. Chandrakantha, Prakash Shetty, Vijesh Nambiyar, Nishitha Isloor, Arun M. Isloor, European Journal of Medicinal Chemistry 45 (2010) 1206-1210.

[8] Samir Bondock, Shymaa Adel, Hassan A. Etman, Farid A. Badria; European Journal of Medicinal Chemistry 48 (2012) 192-199.

[9] Rajesh A. Rane, Pavankumar Bangalore, Sheetal D. Borhade, Preeti K. Khandare, European Journal of Medicinal Chemistry 70 (2013) 49-58.

[10] Jansen M., et al., H. J. Med. Chem. 51 (2008) 4430-4448.

[11] P. C. Unangast, G. P. Shrum, D. T. Conner, C. D. Dyer, D. J. Schrier, J. Med. Chem. 35 (1992) 3691-3698.

[12] V. Ravichandran, S. Shalini, K. Sundram, A. Dhanaraj Sokkalingam, European Journal of Medicinal Chemistry 45 (2010) 2791-2797.

[13] Omar F. A., Mahfouz N. M., Rahman, M. A., Eur. J. Med. Chem.Chim. Ther. 31 (1996) 819.

[14] Dipti L. Namera, Jaynt B. Rathod, Rupali H. Maheta, Umed C. Bhoya, International Letters of Chemistry, Physics and Astronomy 10 (2014) 46-54.

[15] Dipti L. Namera, Umed C. Bhoya, International Letters of Chemistry, Physics and Astronomy, 11(2) (2014) 159-166. 